\title{
Pregnant Driver-Associated Motor Vehicle Crashes in North Carolina, 2001-2008
}

\author{
Catherine J. Vladutiu, $\mathrm{PhD}^{1,2}$, Charles Poole, ScD ${ }^{1}$, Stephen W. Marshall, $\mathrm{PhD}^{1,2}$, Carri \\ Casteel, PhD ${ }^{1,2}$, M. Kathryn Menard, MD ${ }^{3}$, and Harold B. Weiss, PhD ${ }^{4}$ \\ ${ }^{1}$ Department of Epidemiology, Gillings School of Global Public Health, University of North \\ Carolina, CB \#7435, Chapel Hill, North Carolina, 27599-7435, USA 2 Injury Prevention Research \\ Center, University of North Carolina, CB \#7505, Chapel Hill, North Carolina, 27599-7505, USA \\ ${ }^{3}$ Department of Obstetrics \& Gynecology, School of Medicine, University of North Carolina, CB \\ \#7570, Chapel Hill, North Carolina, 27599-7570, USA ${ }^{4}$ Injury Prevention Research Unit, \\ Department of Preventive and Social Medicine, Dunedin School of Medicine, University of Otago, \\ P.O. Box 913, Dunedin 9054, New Zealand
}

\section{Abstract}

Background-Motor vehicle crashes are the leading cause of maternal injury-related mortality during pregnancy in the United States, yet pregnant women remain an understudied population in motor vehicle safety research.

\begin{abstract}
Methods-We estimated the risk of being a pregnant driver in a crash among 878,546 pregnant women, $16-46$ years, who reached the $20^{\text {th }}$ week of pregnancy in North Carolina (NC) from 2001-2008. We also examined the circumstances surrounding the crash events. Pregnant drivers in crashes were identified by probabilistic linkage of live birth and fetal death records and state motor vehicle crash reports.
\end{abstract}

\begin{abstract}
Results-During the eight-year study period, the estimated risk of being a driver in a crash was 12.6 per 1,000 pregnant women. Pregnant women at highest risk of being drivers in serious crashes were 18-24 years old ( 4.5 per 1,000; 95\% confidence interval, CI, 4.3, 4.7), non-Hispanic black $(4.8$ per 1,$000 ; 95 \% \mathrm{CI}=4.5,5.1)$, had high school diplomas only $(4.5$ per 1,$000 ; 95 \%$ $\mathrm{CI}=4.2,4.7)$ or some college ( 4.1 per 1,$000 ; 95 \% \mathrm{CI}=3.9,4.4)$, were unmarried (4.7 per 1,000; $95 \% \mathrm{CI}=4.4,4.9)$, or tobacco users $(4.5$ per 1,$000 ; 95 \% \mathrm{CI}=4.1,5.0)$. A high proportion of crashes occurred between 20-27 weeks of pregnancy (45\%) and a lower proportion of crashes involved unbelted pregnant drivers $(1 \%)$ or airbag deployment (10\%). Forty percent of crashes resulted in driver injuries.
\end{abstract}

Conclusions-NC has a relatively high pregnant driver crash risk among the four U.S. states that have linked vital records and crash reports to examine pregnancy-associated crashes. Crash risks were especially elevated among pregnant women who were young, non-Hispanic black,

(C) 2013 Elsevier Ltd. All rights reserved.

Corresponding Author: Catherine J. Vladutiu, PhD, Department of Epidemiology, Gillings School of Global Public Health, University of North Carolina, Chapel Hill, McGavran-Greenberg Hall, CB \#8050, Chapel Hill, NC 27599-8050, T: (919) 951-8089, cvladutiu@unc.edu.

Publisher's Disclaimer: This is a PDF file of an unedited manuscript that has been accepted for publication. As a service to our customers we are providing this early version of the manuscript. The manuscript will undergo copyediting, typesetting, and review of the resulting proof before it is published in its final citable form. Please note that during the production process errors may be discovered which could affect the content, and all legal disclaimers that apply to the journal pertain. 
unmarried, or used tobacco. Additional research is needed to quantify pregnant women's driving frequency and patterns.

\section{Keywords}

motor vehicle; driver; pregnancy; linkage

\section{INTRODUCTION}

In the United States, motor vehicle crashes are the leading cause of maternal injury-related death during pregnancy. ${ }^{1}$ A pregnant occupant crash rate of 13 per 1,000 person-years was estimated from a probability sample of pregnant occupant crashes in the U.S., while a rate of 26 per 1,000 person-years was estimated for non-pregnant women during the same period. ${ }^{2}$ However, the pregnant occupant crash rate is likely an underestimate due to difficulties in capturing cases. Statewide crash surveillance is lacking and administrative databases are limited, as crash records lack information on pregnancy status and vital records lack data on crash history.

A few studies have addressed these limitations by using linked data sources (i.e., police crash reports and vital records) to estimate state-level pregnancy crash risks. ${ }^{3-5}$ These risks (ranging from $1.0 \%$ to $2.8 \%$ ) are better estimates of crashes during pregnancy, but the denominators do not account for variable time spent driving a motor vehicle during pregnancy. A recent study suggested that these estimates may be on the "lower end of the risk spectrum" since they are from states that have older maternal ages at birth and lower overall severe crash risks in older women (i.e., Washington, Pennsylvania) or younger maternal ages and lower severe crash risks among younger women (i.e., Utah). ${ }^{3}$ The pregnancy-associated crash risks in states, such as North Carolina (NC), that have higher severe crash risks among women of reproductive age are unknown. ${ }^{3}$

Pregnant women are an understudied population in motor vehicle safety research, thus little is known about the characteristics of pregnant drivers in crashes and the circumstances surrounding these crash events. This information is important for informing the development of crash prevention strategies. Our objectives were to use linked data sources in NC to estimate the overall risk of being a pregnant driver in a crash, to estimate crash risks by selected maternal characteristics, and to describe the characteristics of pregnant driverassociated crashes among pregnant women, aged 16-46 years, with singleton pregnancies.

\section{METHODS}

\subsection{Study population}

This study included a cohort of 878,546 pregnant NC residents, 16-46 years, who reached the $20^{\text {th }}$ week of pregnancy and delivered a singleton infant in NC between January 1, 2001 and December 31, 2008 (Figure 1). These women were identified from live birth and fetal death records from the NC State Center for Health Statistics ( $n=993,274)$. We removed records for pregnant women aged less than 16 years at the time of delivery $(n=7,075)$ because driver crashes in this pre-licensure age group are uncommon. We also excluded women older than 46 years at delivery $(n=237)$, and those with multiple gestation pregnancies $(n=33,360)$. Additional records were excluded $(n=603)$ if there were missing data for at least one of the following: mother's age, multiple gestation status or gestational age at delivery. Records were also excluded for 73,453 pregnant women who did not meet at least one of the cohort inclusion criteria. 


\subsection{Probabilistic record linkage}

To identify women who were pregnant drivers in crashes, individual vital records were probabilistically linked to state crash records using the following match fields and comparison methods for assigning an agreement weight: mother's first and last name (30\% prefix difference allowed), middle name (exact match), date of birth (exact match), race (exact match), and residential county (exact match) (Figure 1). Linked record pairs were ranked from highest to lowest based on their match weights and probabilities. We specified a false positive rate of 0.01 (or 1\%) for the record linkage prior to the study. Matched pairs were selected one-by-one until the desired false positive rate was obtained. There were 103,713 linked vital records and crash reports of which 1,037 (1\%) were considered false matches. This linkage was performed using LinkSolv generalized linkage software (Strategic Matching Inc., Morrisonville, NY, 2009). After the linkage was completed, the date of the last menstrual period (LMP), gestational age, and the crash date were compared to ensure that the crash occurred during pregnancy. There were 26,913 linked records with crash events that occurred during pregnancy.

\subsection{Measures}

2.3.1 Motor vehicle crashes and crash severity-Motor vehicle crashes involved a NC licensed female driver of a motor vehicle or passenger truck beyond the $20^{\text {th }}$ week of pregnancy. Crashes are reported by police when they occur on a public roadway and result in a fatality or non-fatal personal injury to any vehicle occupant, total property damage greater than $\$ 1000$, or property damage of any amount to a vehicle seized. Only crashes involving a NC licensed driver were included because identifiers from the driver license records were needed for the linkage and identifying information for occupants is not available from crash reports.

Crash severity was assessed by police-reported vehicle damage ratings as determined by the direction of impact, type of impact, and damage location. ${ }^{6}$ Severity ratings ranged from 0 (no damage) to 7 (severe damage). For this study, serious or severe crashes were defined as those with a vehicle damage rating of at least 3 (i.e., crashes that resulted in more than minor dents or gouges, such as crumpling of sheet metal and/or deformation of the structure or frame).

2.3.2 Maternal characteristics-Maternal characteristics included gestational age, maternal age, maternal race, Hispanic ethnicity, maternal education, marital status, prenatal tobacco use, prenatal alcohol use, prenatal care before the $20^{\text {th }}$ week, and parity (defined as the total number of prior live births). Gestational age was estimated using the National Center for Health Statistics' method for estimating gestational age in the U.S. vital statistics. ${ }^{7,8}$ Estimates of gestational age relied primarily on LMP, but were edited if necessary. If the LMP date was missing or provided an implausible gestational age (i.e., based on comparing weeks of gestation with birth weight, $n=51,593$ or $5.2 \%$ ), the clinical estimate was used. If the LMP date and clinical estimate were both missing $(\mathrm{n}=531,<0.1 \%)$, the physician's estimate (i.e., estimated from pregnancy history, early ultrasound, or examination of the stillborn infant), which is only reported on fetal death records, was used. There were 481 records $(<0.1 \%)$ missing values for all measures of gestational age. Race and Hispanic ethnicity were combined into one measure with four categories, including nonHispanic (NH) white, NH black, other NH race (i.e., American Indian, Asian, Pacific islander), and Hispanic. Data for these maternal characteristics were obtained from vital records.

2.3.3 Crash characteristics-Crash information included police-reported driver characteristics (i.e., suspected alcohol use at the time of the crash, driver injury, seat belt 
use), vehicle characteristics (i.e., airbag deployment, estimated speed at impact, vehicle type, number of occupants), and environmental characteristics (i.e., ambient light, crash locality, road surface, and weather). Driver injury was reported by the investigating police officer using a five-point injury severity scale (i.e., KABCO) ${ }^{9}$ Gestational age at the time of the crash was estimated by comparing the date of the crash to the date of delivery.

\subsection{Statistical analysis}

We used binomial regression to model the risk of being a pregnant driver in a crash after the $20^{\text {th }}$ week of pregnancy and to estimate crash risks for selected maternal characteristics. Since it was impossible to obtain an accurate denominator of pregnancies before 20 weeks due to the lack of information for early fetal losses and terminations in vital records, only pregnancies completing the $20^{\text {th }}$ week and crashes occurring after the $20^{\text {th }}$ week were counted in the analyses. Gestational age-specific crash risks were estimated as the number of pregnant drivers in crashes during two-week periods divided by the total number of women who were pregnant at the beginning of that period. We also conducted descriptive analyses to examine the crash characteristics. This study was approved by the Institutional Review Board of the University of North Carolina at Chapel Hill.

\section{RESULTS}

\subsection{Study population}

A total of 878,546 pregnant women met the eligibility criteria in 2001-2008. High proportions of these women were 25-34 years old, non-Hispanic white, educated at least through high school, married, began prenatal care before the $20^{\text {th }}$ week of pregnancy, did not use tobacco or alcohol during pregnancy, and had at least one prior live birth (Table 1). The mean number of weeks of pregnancy completed by women in this cohort was 38.7 weeks (standard deviation=2.7); the median was 39.0 weeks.

There were 11,087 pregnant women (12.6 per 1,000 pregnant women) who were drivers in at least one crash after the $20^{\text {th }}$ week of pregnancy; 3,217 were in at least one serious or severe crash (3.7 per 1,000 pregnant women) and 7,936 were in at least one non-severe crash (9.0 per 1,000 pregnant women) (Table 1). Nearly all (98\%) pregnant drivers in a crash were involved in only one crash $(\mathrm{n}=10,931) ; 153$ were involved in two, and 3 in three crashes.

\subsection{Maternal characteristics and crash risks}

Pregnant women at highest risk of being drivers in a serious or severe crash were 18-24 years, NH black, with high school diplomas only or some college, unmarried, or used tobacco (Table 1). The lowest severe crash risks were among those who were 16-17 years old, 35 years or older, or Hispanic. Maternal characteristics for non-severe crashes were similar, with the exception of prenatal care where low non-severe crash risks were estimated for women who delayed prenatal care initiation.

Gestational age-specific driver crash risks remained relatively constant at 1.5 per 1,000 pregnant women (standard error, SE, 0.04) from 20-31 weeks of pregnancy (Figure 2). The driver crash risk declined after 31 weeks with the lowest risk ( 0.04 per 1,000 pregnant women, $\mathrm{SE}=0.02$ ) estimated between $40-42$ weeks.

\subsection{Crash characteristics}

There were 11,246 crashes that occurred after the $20^{\text {th }}$ week of pregnancy among pregnant women in the study population (11,087 pregnant women were in one or more crashes and each crash was counted separately for this analysis). A high proportion of crashes involved 
pregnant drivers who were between 20-27 weeks of pregnancy, not under the influence of alcohol, or were driving at low speeds, in passenger cars, and with no occupants (Table 2). Fewer crashes involved unbelted pregnant drivers, resulted in driver injuries, or initiated airbag deployment. Most crashes occurred during the day, in urban areas, on dry roads, and during clear weather conditions.

\section{DISCUSSION}

Previous state-level linkage studies have estimated pregnancy crash risks that ranged from $1.0 \%$ among pregnant front seat occupants in Washington State ${ }^{4}$ to $2.8 \%$ among pregnant drivers in Utah. ${ }^{5}$ A recent study in Pennsylvania ${ }^{3}$ reported a crash risk of $1.1 \%$ among pregnant drivers. However, these studies counted crashes that occurred at any detectable time during pregnancy, despite the undercount of pregnancies at risk early in pregnancy, owing to the high frequency of fetal loss during that period and the lack of vital records reporting early losses and terminations. Therefore, a direct comparison of our results with the estimated crash risks from these other studies is difficult due to differences in methodology. However, if we had calculated our driver crash risk using the same method as these other studies (i.e., by counting all crashes that occurred during pregnancy, including those occurring before the $20^{\text {th }}$ week), then $\mathrm{NC}$ would have a reported pregnant driver crash risk of $2.9 \%(25,168 / 878,546)$.

We identified variations in the pregnant driver crash risk by maternal characteristics, such that the risk of being in a crash was higher for women who were young (i.e., 18 to 24 years), black, moderately educated, unmarried, or used tobacco. Similarly, previous research found a higher proportion of women who crashed during pregnancy to be younger, ${ }^{5,10}$ nonwhite, ${ }^{10}$ less educated, ${ }^{10}$ unmarried, ${ }^{10}$ and tobacco users ${ }^{5,10}$ than pregnant women who were not in crashes. We also found that pregnant women in our study population were at lower risk of being drivers in crashes during the last few weeks of pregnancy. Much of this decline may be attributed to a lower frequency of driving during that time or other modifications of driving behavior. Hispanic women were also at lower risk of being drivers in crashes, regardless of crash severity. We expected higher crash risks for these women since Hispanics, in general, have an increased involvement in crashes. ${ }^{11}$ Although severe crash risks did not vary by prenatal care initiation, pregnant women who delayed prenatal care initiation were at lower risk of being in a non-severe crash. Based on previous research, we expected driver crash risks to be consistently higher among pregnant women who delayed care. $^{10}$

This study expands on previous literature by estimating the pregnant driver crash risk in a state that is known to have a relatively high severe crash risk among reproductive-aged women. The strengths of this study include the large size and diversity of the cohort enumerated over an eight-year study period, the use of record linkage to ascertain crashes during pregnancy (since these are not routinely documented), and the examination of robust crash characteristics from NC's comprehensive crash reports. As compared to previous studies, this study provides the most valid risk estimate for pregnant driver crashes occurring after the $20^{\text {th }}$ week of pregnancy.

This study has several limitations. Similar to previous studies, we were unable to quantify the amount of time or miles spent driving during pregnancy and cannot confine the denominator of our estimates to person-time spent driving. We instead estimated the number of women at risk of being a driver in a crash among all pregnant women, aged 16-46, with singleton pregnancies, who completed the $20^{\text {th }}$ week of pregnancy, regardless of how much they drove. Additionally, this study only included crashes that occurred among licensed drivers, yet we could not determine the license status of all pregnant women in our study 
population. Based on a crude estimation of licensed drivers in NC, approximately $7 \%$ of NC female residents aged 16 to 44 are unlicensed. ${ }^{12,13}$ If we assume that a small proportion (up to $7 \%$ ) of women in our study population were not at risk of being a licensed driver in a crash, then the reported crash risks may be slightly underestimated. We were also unable to observe pregnancies that ended before the $20^{\text {th }}$ week, women who moved out of NC during pregnancy, out-of-state crashes, and pregnant driver crashes that did not link to vital records. Although most pregnant women who are involved in crashes are drivers ${ }^{2}$, our study, like most prior research, could not identify women who were passengers in crashes.

There is the potential for misclassification of key measures from the linked data sources. Gestational age was used to determine whether a crash occurred during pregnancy and after completion of the $20^{\text {th }}$ week, but LMP-based and clinical estimates of gestational age are susceptible to error in vital records. ${ }^{7,14-16} \mathrm{We}$ addressed this limitation by creating a composite measure of gestational age that utilized both sources of information, but misclassified estimates likely remain for some records. Previous studies have also found that behavioral measures, such as prenatal tobacco and alcohol use, are unreliable in vital records and may be underreported. ${ }^{17,18}$ Hence, we urge caution when interpreting the results that include these behavioral measures. Similar to previous studies, ours also lacks information on the instantaneous change in velocity (i.e., delta V), which could provide a more valid measure of crash severity.

\section{CONCLUSION}

As one of only four U.S. states that have used linked records to quantify the risk of being a pregnant driver in a crash, $\mathrm{NC}$ has a relatively high pregnant driver crash risk. This risk may be even higher in other states, particularly those with crash risks among reproductive-aged women that are higher than in NC (e.g., Mississippi, Montana, Wyoming). To further increase awareness of this important public health issue and to identify states with potentially higher pregnancy-related crash risks, we could improve our ability to track crashes during pregnancy. Although existing databases estimate pregnancy-associated crashes, including the National Highway Traffic Safety Administration's Fatality Analysis Reporting System (FARS) and National Automotive Sampling System (NASS), they likely underestimate these events. In the absence of a reliable data system for tracking crashes during pregnancy, more states could adopt pregnancy-related crash surveillance systems by utilizing record linkage procedures, such as those developed through the Crash Outcome Data Evaluation System (CODES), which links crash reports to other injury outcome databases. ${ }^{19}$

Crash risks were especially elevated among pregnant women who were young, black, less than college educated, or unmarried. Thus, health care providers should use this information to educate their patients about the risk of being a driver in a crash during pregnancy, particularly women with these characteristics. Unfortunately, little is known about driving patterns during pregnancy. Additional information on driving frequency and patterns would enable the estimation of more valid crash risks, particularly those at different weeks of pregnancy.

\section{Acknowledgments}

CV received partial support from grant T32-HD052468-03 from the Eunice Kennedy Shriver National Institute of Child Health and Human Development, National Institutes of Health, Bethesda, Maryland. The authors would like to thank Larry Cook at the University of Utah for his assistance with the probabilistic linkage, Eric Rodgman and Carol Martell at the University of North Carolina Highway Safety Research Center for their help with crash data acquisition and preparation, and Matt Avery at the North Carolina State Center for Health Statistics for his help with vital records data acquisition and preparation. 


\section{References}

1. Rochat RW, Koonin LM, Atrash HK, Jewett JF. Maternal mortality in the United States: report from the Maternal Mortality Collaborative. Obstet Gynecol. 1988; 72(1):91-97. [PubMed: 3380512]

2. Weiss H, Strotmeyer S. Characteristics of pregnant women in motor vehicle crashes. Inj Prev. 2002; 8:207-210. [PubMed: 12226117]

3. Weiss H, Sauber-Schatz E, Herring A. Motor-vehicle crashes during pregnancy: a retrospective cohort study. Open J Obstet Gynecol. 2011; 1(4):202-207.

4. Schiff MA, Mack CD, Kaufman RP, Holt VL, Grossman DC. The effect of air bags on pregnancy outcomes in Washington State: 2002-2005. Obstet Gynecol. 2010; 115(1):85-92. [PubMed: 20027039]

5. Hyde L, Cook L, Olson L, Weiss H, Dean J. Effect of motor vehicle crashes on adverse fetal outcomes. Obstet Gynecol. 2003; 102(2):279-286. [PubMed: 12907100]

6. University of North Carolina Highway Safety Research Center. Vehicle damage scale for traffic accident investigators. 3. National Safety Council; 1984.

7. Martin JA. United States vital statistics and the measurement of gestational age. Paediatr Perinat Epidemiol. 2007; 21 (Suppl 2):13-21. [PubMed: 17803614]

8. Wier ML, Pearl M, Kharrazi M. Gestational age estimation on United States live birth certificates: a historical overview. Paediatr Perinat Epidemiol. 2007; 21 (Suppl 2):4-12. [PubMed: 17803613]

9. Manual on Classification of Motor Vehicle Traffic Accidents Seventh Edition-Revision of ANSI D-16.1-1996. Itasca, IL: National Safety Council; 2007.

10. Schiff M, Holt V. Pregnancy outcomes following hospitalization for motor vehicle crashes in Washington State from 1989 to 2001. Am J Epidemiol. 2005; 161:503-510. [PubMed: 15746466]

11. Sainz S, Saito M. Hispanic involvement in motor vehicle accidents. Transportation Research Board Record. 1996; 1560:27-33.

12. Federal Highway Administration. [Accessed January 9, 2013] Highway Statistics. 2001-2008. http://www.fhwa.dot.gov/policyinformation/quickfinddata/qfdrivers.cfm

13. United States Census Bureau. [Accessed January 9, 2013] Population Estimates. 2011. http:// www.census.gov/popest/estimates.html

14. Ananth CV. Menstrual versus clinical estimate of gestational age dating in the United States: temporal trends and variability in indices of perinatal outcomes. Paediatr Perinat Epidemiol. 2007; 21 (Suppl 2):22-30. [PubMed: 17803615]

15. David RJ. The quality and completeness of birthweight and gestational age data in computerized birth files. Am J Public Health. 1980; 70(9):964-973. [PubMed: 7406096]

16. Dietz PM, England LJ, Callaghan WM, Pearl M, Wier ML, Kharrazi M. A comparison of LMPbased and ultrasound-based estimates of gestational age using linked California livebirth and prenatal screening records. Paediatr Perinat Epidemiol. 2007; 21 (Suppl 2):62-71. [PubMed: 17803619]

17. Vinikoor LC, Messer LC, Laraia BA, Kaufman JS. Reliability of variables on the North Carolina birth certificate: a comparison with directly queried values from a cohort study. Paediatr Perinat Epidemiol. 2010; 24(1):102-112. [PubMed: 20078836]

18. Buescher P, Taylor K, Davis M, JMB. The quality of the new birth certificate data: a validation study in North Carolina. Am J Public Health. 1993; 83(8):1163-1165. [PubMed: 8342728]

19. National Highway Traffic Safety Administration. [Accessed January 9, 2013] State Data Program \& CODES. http://www.nhtsa.gov/Data/State+Data+Program+\&+CODES 


\section{Highlights}

- North Carolina has a relatively high pregnant driver crash risk (12.6 per 1,000 pregnant women).

- Crash risks were especially elevated among pregnant women who were young, black, moderately educated, or unmarried.

- Pregnant women were at lower risk of being drivers in crashes during the last few weeks of pregnancy.

- More states could adopt pregnancy-related crash surveillance systems by utilizing record linkage procedures.

- More research is needed to quantify pregnant women's driving frequency and patterns. 


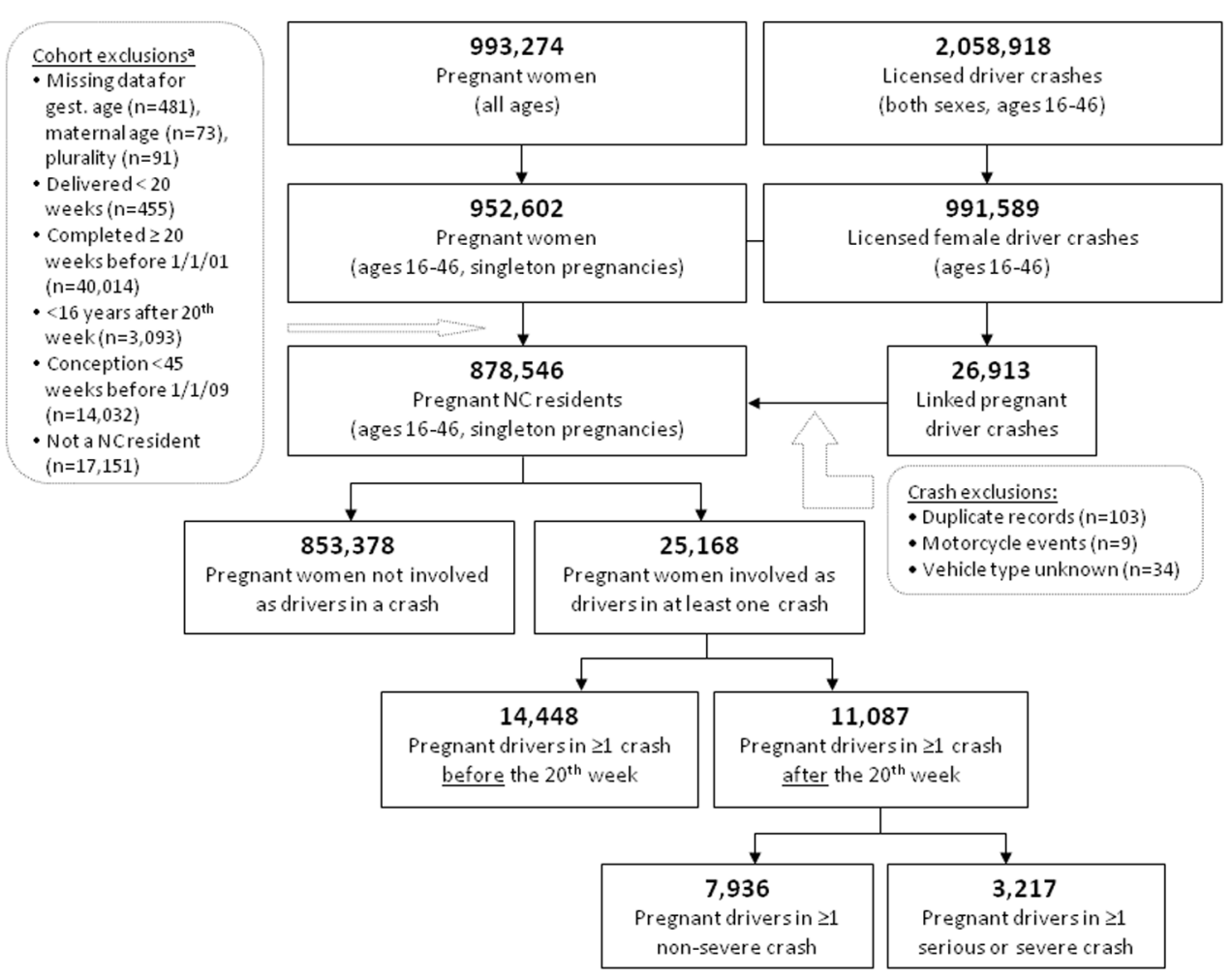

Figure 1.

Flow chart to estimate the number of pregnant drivers who were in motor vehicle crashes, by crash severity, after the $20^{\text {th }}$ week of pregnancy in North Carolina, 2001-2008. ${ }^{\text {a }}$ The criteria are not mutually exclusive, thus some records are counted in multiple categories. 


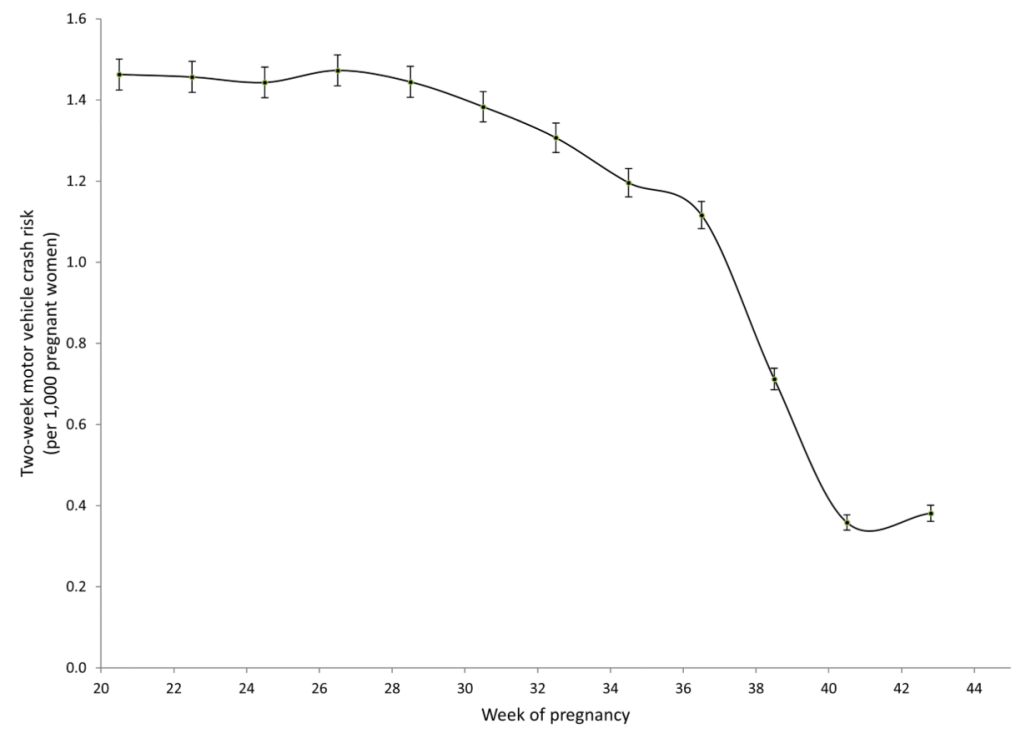

Figure 2.

The risk of being a driver in a motor vehicle crash after the $20^{\text {th }}$ week, by week of pregnancy, among pregnant women in North Carolina ( $\mathrm{N}=878,546), 2001-2008$. The mean number of weeks of pregnancy completed by women in our cohort was 38.7 weeks (standard deviation=2.66); the median was 39.0 weeks. 


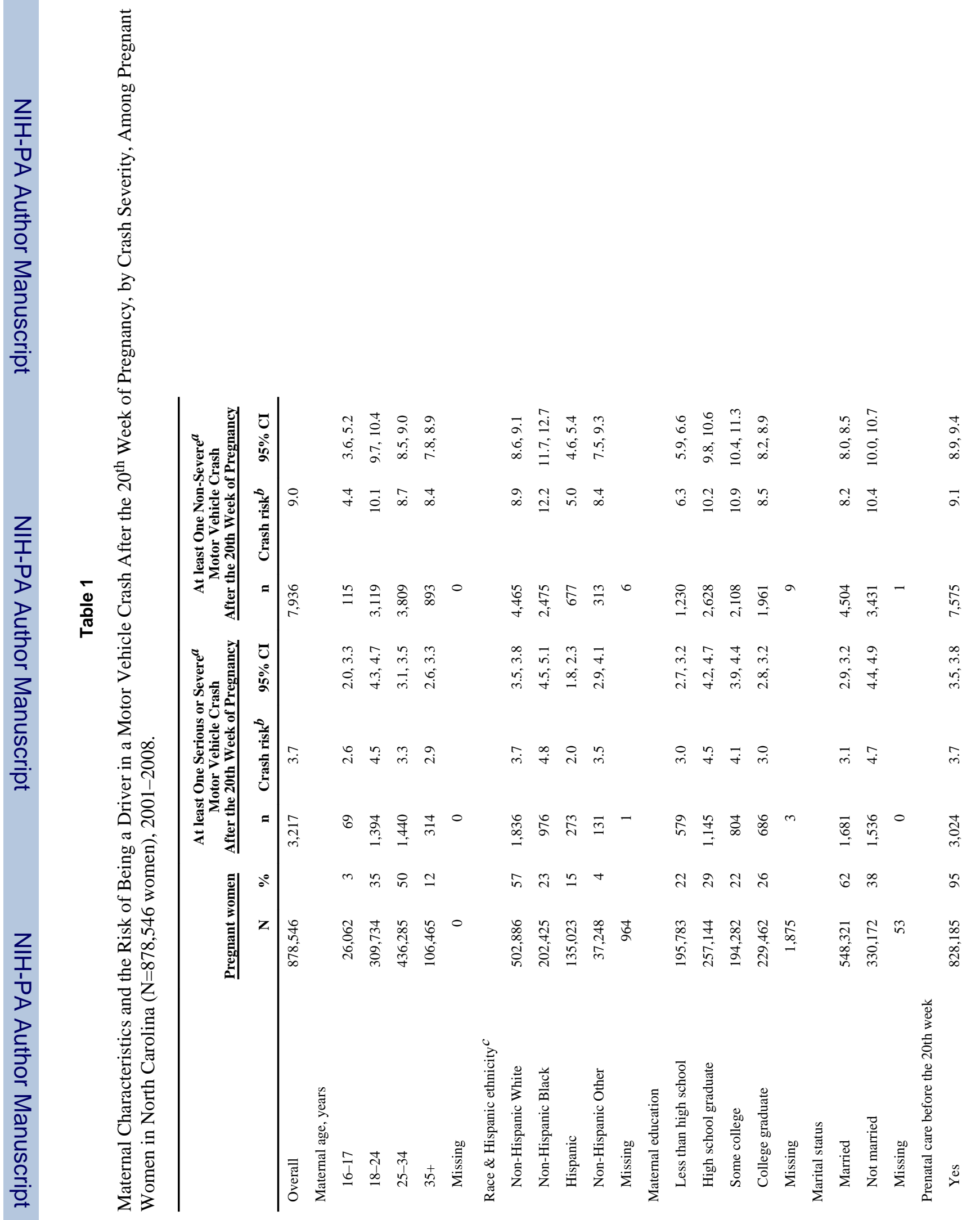




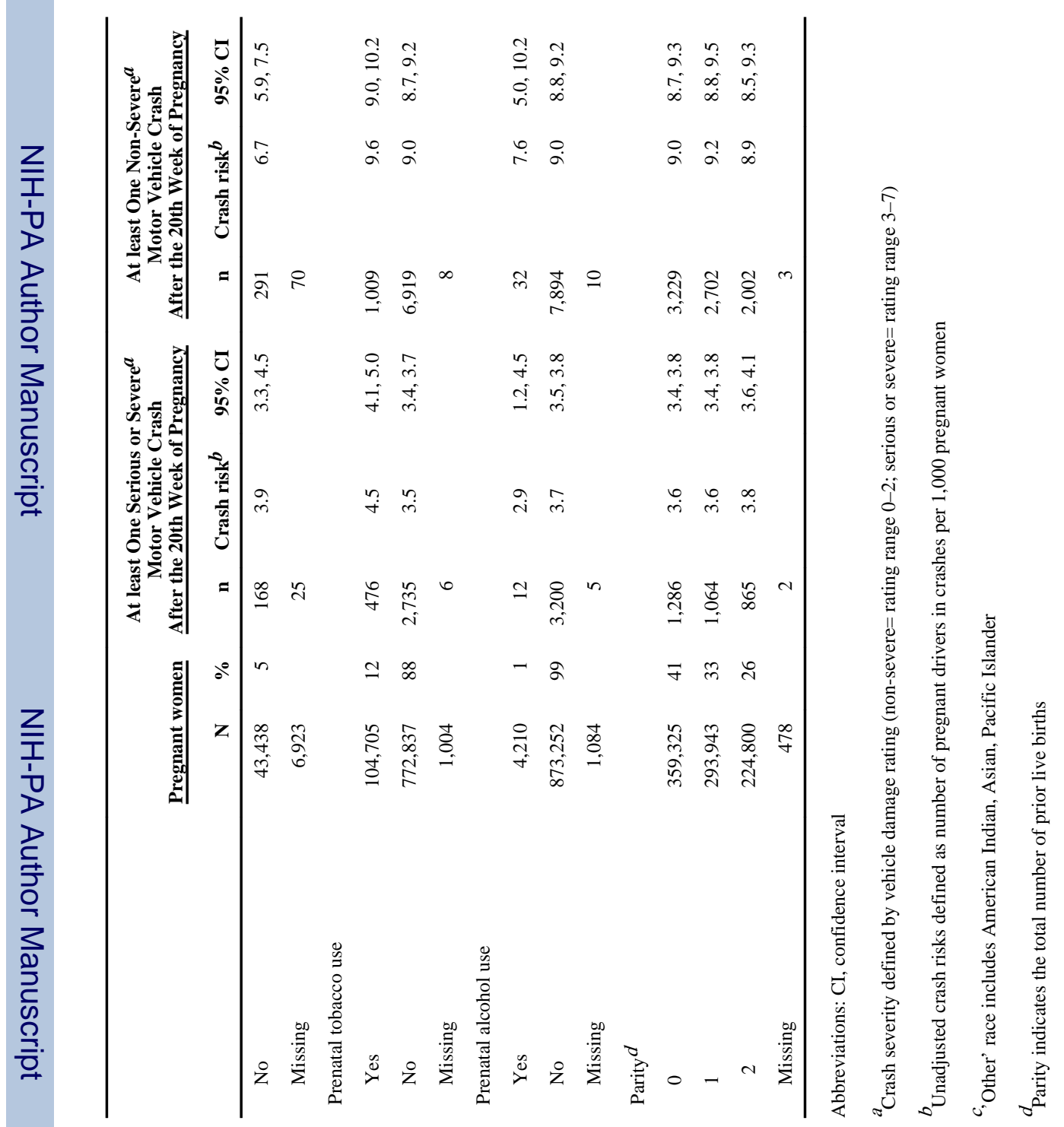


Table 2

Characteristics of Pregnant Driver-Associated Crashes After the 20 th Week of Pregnancy, By Crash Severity, in North Carolina ( $\mathrm{N}=11,246$ crashes), 2001-2008

\begin{tabular}{|c|c|c|c|c|}
\hline & \multicolumn{2}{|c|}{$\begin{array}{c}\text { Serious or Severe }{ }^{a} \\
\text { Motor Vehicle Crashes }(\mathrm{N}=\mathbf{3 , 2 2 4}) \\
\end{array}$} & \multicolumn{2}{|c|}{ 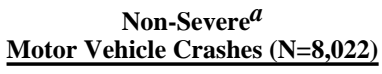 } \\
\hline & $\mathbf{n}$ & $\%$ & $\mathbf{n}$ & $\%$ \\
\hline \multicolumn{5}{|l|}{ Driver characteristics } \\
\hline \multicolumn{5}{|c|}{ Gestational age at time of crash } \\
\hline $20-27$ & 1,483 & 46 & 3,626 & 45 \\
\hline $28-32$ & 846 & 26 & 2,163 & 27 \\
\hline $33-36$ & 593 & 18 & 1,474 & 18 \\
\hline $37+$ & 302 & 9 & 759 & 10 \\
\hline Missing & 0 & 0 & 0 & 0 \\
\hline \multicolumn{5}{|c|}{ Suspected alcohol use at time of crash } \\
\hline Yes & 23 & 1 & 25 & 1 \\
\hline No & 3,201 & 99 & 7,997 & 99 \\
\hline Missing & 0 & 0 & 0 & 0 \\
\hline \multicolumn{5}{|l|}{ Seat belt use } \\
\hline None & 68 & 2 & 102 & 1 \\
\hline Belt & 3,082 & 96 & 7,772 & 97 \\
\hline Missing & 74 & 2 & 148 & 2 \\
\hline \multicolumn{5}{|l|}{ Driver injury } \\
\hline None & 1,326 & 41 & 5,312 & 66 \\
\hline Possible & 1,469 & 46 & 2,430 & 30 \\
\hline Non-Disabling & 336 & 10 & 140 & 2 \\
\hline Disabling or Fatal & 34 & 1 & 5 & $<1$ \\
\hline Missing & 59 & 2 & 135 & 2 \\
\hline \multicolumn{5}{|l|}{ Vehicle characteristics } \\
\hline \multicolumn{5}{|l|}{ Airbag deployment } \\
\hline No airbag & 534 & 16 & 1,358 & 17 \\
\hline Not deployed & 1,869 & 58 & 6,282 & 78 \\
\hline Deployed & 792 & 25 & 320 & 4 \\
\hline Missing & 29 & 1 & 62 & 1 \\
\hline \multicolumn{5}{|l|}{ Speed at impact } \\
\hline$<25 \mathrm{mph}$ & 1,288 & 40 & 4,618 & 58 \\
\hline $25-45 \mathrm{mph}$ & 1,433 & 44 & 2,050 & 25 \\
\hline$>45 \mathrm{mph}$ & 257 & 8 & 329 & 4 \\
\hline Missing & 246 & 8 & 1,025 & 13 \\
\hline \multicolumn{5}{|l|}{ Vehicle type } \\
\hline Passenger car & 3,212 & 99 & 7,953 & 99 \\
\hline Other & 12 & 1 & 69 & 1 \\
\hline Missing & 0 & 0 & 0 & 0 \\
\hline
\end{tabular}




\begin{tabular}{|c|c|c|c|c|}
\hline & \multicolumn{2}{|c|}{$\begin{array}{c}\text { Serious or Severe }{ }^{a} \\
\text { Motor Vehicle Crashes ( } N=3,224)\end{array}$} & \multicolumn{2}{|c|}{$\begin{array}{c}\text { Non-Severe }^{a} \\
\text { Motor Vehicle Crashes (N=8,022) }\end{array}$} \\
\hline & $\mathbf{n}$ & $\%$ & $\mathbf{n}$ & $\%$ \\
\hline 1 (driver only) & 1,830 & 57 & 4,811 & 60 \\
\hline 2 & 792 & 25 & 1,883 & 23 \\
\hline $3+$ & 602 & 19 & 1,328 & 17 \\
\hline Missing & 0 & 0 & 0 & 0 \\
\hline \multicolumn{5}{|l|}{ Environmental characteristics } \\
\hline \multicolumn{5}{|l|}{ Ambient light } \\
\hline Daylight & 2,539 & 79 & 6,522 & 81 \\
\hline Dark & 681 & 21 & 1,489 & 19 \\
\hline Missing & 4 & $<1$ & 11 & $<1$ \\
\hline \multicolumn{5}{|l|}{ Crash locality } \\
\hline Rural ( $<30 \%$ developed) & 867 & 27 & 1,443 & 18 \\
\hline Mixed (30-70\% developed) & 556 & 17 & 1,184 & 15 \\
\hline Urban (>70\% developed) & 1,801 & 56 & 5,395 & 67 \\
\hline Missing & 0 & 0 & 0 & 0 \\
\hline \multicolumn{5}{|l|}{ Road surface } \\
\hline Dry & 2,601 & 81 & 6,587 & 82 \\
\hline Wet & 560 & 17 & 1,321 & 16 \\
\hline Snow or Ice & 50 & 2 & 86 & 1 \\
\hline Other & 5 & $<1$ & 7 & $<1$ \\
\hline Missing & 8 & $<1$ & 21 & $<1$ \\
\hline \multicolumn{5}{|l|}{ Weather condition } \\
\hline Clear & 2,261 & 70 & 5,722 & 71 \\
\hline Cloudy & 637 & 20 & 1,555 & 19 \\
\hline Rain or Snow & 321 & 10 & 740 & 9 \\
\hline Other & 5 & $<1$ & 5 & $<1$ \\
\hline Missing & 0 & 0 & 0 & 0 \\
\hline
\end{tabular}

${ }^{a}$ Crash severity defined by vehicle damage rating (non-severe $=$ rating range $0-2$; serious or severe $=$ rating range $3-7$ ) 